\title{
A Reliable Visual Inspection Method for Vulnerability Assessment of Hyperstatic Structures Using Fuzzy Logic Analysis
}

\author{
Maria Valeria Piras, ${ }^{1}$ Fausto Mistretta, ${ }^{1}$ Maria Luisa Fadda, ${ }^{1}$ and Luisa Deias ${ }^{2}$ \\ ${ }^{1}$ Department of Civil Engineering, Environmental Engineering and Architecture (DICAAR), University of Cagliari, \\ Via Marengo 2, 09123 Cagliari, Italy \\ ${ }^{2}$ Department of Electrical and Electronic Engineering (DIEE), University of Cagliari, Via Marengo 2, 09123 Cagliari, Italy
}

Correspondence should be addressed to Maria Valeria Piras; mvpiras@unica.it

Received 20 February 2015; Revised 30 June 2015; Accepted 7 July 2015

Academic Editor: F. Pacheco-Torgal

Copyright (C) 2015 Maria Valeria Piras et al. This is an open access article distributed under the Creative Commons Attribution License, which permits unrestricted use, distribution, and reproduction in any medium, provided the original work is properly cited.

Fuzzy logic applied to the visual inspection of existing buildings has been proposed in relation to simple structures. Isostatic structures are characterized by a unique and known collapse mechanism, which does not vary with geometry or load change. In this paper we apply fuzzy logic to visual inspection for complex structures such as hyperstatic ones in which the collapse mechanism depends not only on the geometry but also on the size and disposition of loads. The goal of this paper is to give relevant weight, in the fuzzy analysis, not only to the single expression of degradation, due to its localization within the element, but also to the structural element itself by assigning a different resistance to the various elements. The underlying aim of the proposed method is to manage, evaluate, and process all the information coming from visual inspections in order to realize a management information system for the evaluation of the safety level of even complex structures.

\section{Introduction}

In recent years the need for a reliable evaluation of safety for existing buildings has become ever more necessary [1].

This request derives both from traumatic events that have caused great impact on the population and also from greater attention paid by public administrations in relation to the recovery of buildings and the need to preserve constructions holding a strategic and functional importance.

In order to formulate an objective judgment on the safety level $[2,3]$ of existing structures we can identify five operations (not all of them strictly necessary):

(i) Historical data collection.

(ii) Visual inspection.

(iii) In situ (nondestructive) tests.

(iv) Lab test (on properly chosen samples).

(v) In situ (destructive) tests.
Through a comparative critical examination of all the information collected, a diagnosis on the degradation level of the structure under observation can be produced.

However, often the only available diagnosis instrument, in order to evaluate the vulnerability of a construction and decide if further damage evaluation with other tools is required, is represented by visual inspections, mainly because of the need to restore safety conditions in a short time.

Thus, visual inspections become the ruling practice in the management of maintenance, even when the number and importance of the constructions are significant.

The process of evaluation of degradation on the basis of the results of visual inspection is heavily affected by subjectivity. The staff in charge of the inspection write down on a safety assessment card a linguistic statement, which represents the subjective judgment for the degradation under examination. When relying only on visual inspection both the problems of dealing with different levels of expertise 
of the inspectors and the problems of handling subjective information on degradation raise this information, expressed by means of linguistic statements, which needs to be turned into objective and reliable assessments.

In order to use visual inspection as a robust and reliable instrument to evaluate the safety level of a construction it was decided to take advantage of the ability of fuzzy logic to treat uncertainty as expressed by linguistic judgments $[4,5]$. The information undergoes the three steps of fuzzification, inference, and defuzzification [4, 6-11].

Thanks to the key role played by inference, where the technical knowledge of experts is taken into account, fuzzy logic is then able to output an objective evaluation of the safety level of a construction, based only on visual inspection.

This method provides a quick, low cost, and reliable way of assessing the evolution of degradation in a structure [1215].

\section{Fuzzy Logic}

Fuzzy logic was introduced in 1965 [4, 6-11] as a mathematical theory for dealing with uncertainties expressed using language and is now universally recognized as a highly effective mathematical tool for resolving a wide range of problems characterized with nonprobabilistic uncertainty. Fuzzy logic is innovative: it revolutionizes the classical theory of sets and the semantic concept of truth. We can find a variety of applications in civil engineering [16-20].

According to the traditional definition of "set," an element can belong to a set or not. According to the fuzzy set theory an element belongs to a set with a certain degree of membership [4-11].

Similarly, in classical logic a statement is evaluated as "true" or "false" while fuzzy logic assigns a value of partial truth. All this is particularly suitable for those situations where there can be no absolute certainty about a phenomenon. It is useful to specify that such uncertainty can be referred to the judgment of the operator in estimating the phenomenon or to the phenomenon itself.

There are three basic steps of a generic fuzzy process: fuzzification, inference, and defuzzification (Figure 1).

The fuzzification procedure consists in transforming the numerical value of the considered variable in its corresponding value of membership to the given fuzzy sets through the corresponding membership function.

The membership functions typically overlap so that values of the variable can partially belong to multiple fuzzy sets. The wider the area that overlaps, the more the uncertainty the system includes.

The procedure of inference involves the application of the rules of combination of fuzzy sets. Usually these are simple linguistic expressions, which are converted to mathematical formalism in the language of the "if ... then" logic. This is important because information gathered through the examination of a given problem can be used without any translation into formulas, which are often of complex determination.

The output is also a fuzzy membership value that can be used either "raw" as qualitative assessment or defuzzified as a real number, compatible with nonfuzzy approaches [4-11].

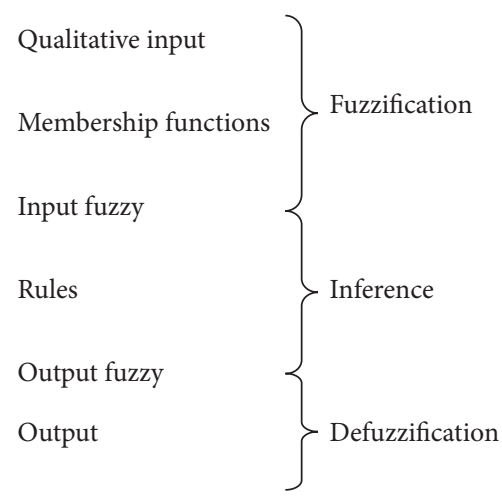

FIGURE 1: Fuzzy-system architecture.

\section{Safety Assessment}

The aim of this research is to determine, through only the process of visual inspection, the probability of failure $P_{f}$ of an existing building in relation to the state of degradation and to the referred limit state [12-15, 21].

The value of the safety assessment is well specified by Eurocode $[22,23]$ as $P_{f}=10^{-n}$, where $P_{f}$ is the failure probability and the safety exponent $n$ can assume values between 1 and 7 .

In this study we will refer to the Ultimate Limit State (ULS), which is the limit state associated with collapse or other similar forms of structural failure. The safety assessment is positive if

$$
P_{f} \leq P_{f}^{*}
$$

This inequality indicates the possibility that the state limit defined above is reached. According to Eurocode the probability $P_{f}^{*}$ that a structure in 50 years will reach the Ultimate Limit State is equal to $P_{f}^{*}=10^{-4}$, and (1) can be rewritten as

$$
P_{f} \leq 10^{-4} ; \quad n \geq 4 .
$$

This value represents the limit value $P_{f}^{*}$ which must not be exceeded by $P_{f}$ of the structure so that the safety assessment is positive.

\section{Application of Fuzzy Logic to Hyperstatic Structures}

Fuzzy logic applied to the visual inspection of existing buildings has been proposed in relation to simple structures such as isostatic structures [12-15]. In this research we have taken into account the weight of the event of deterioration in relation to the limit state and the localization of the damage within the structural element [24-27].

In this paper we present the results of the application of fuzzy logic to hyperstatic structures.

In particular we chose as a case study the one shown in Figures 2 and 3, a frame type with bidimensional spans of 


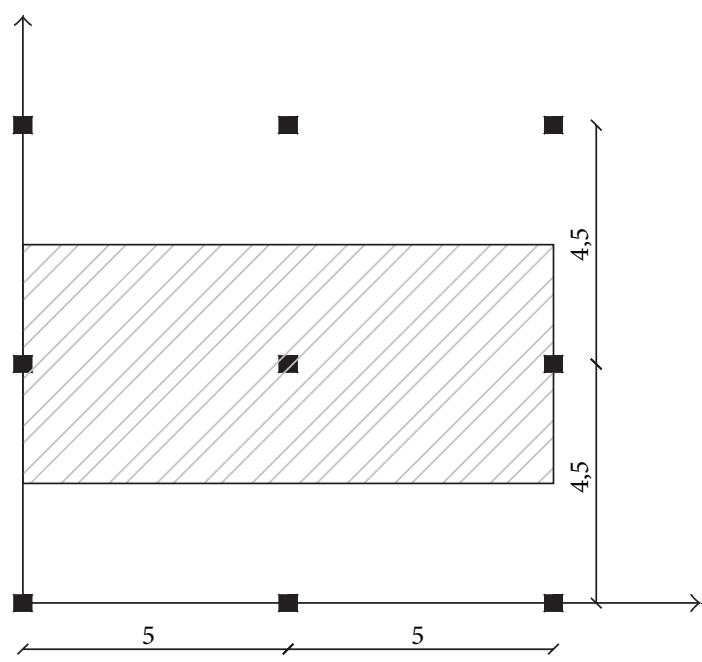

Figure 2: Plan view hyperstatic structure.

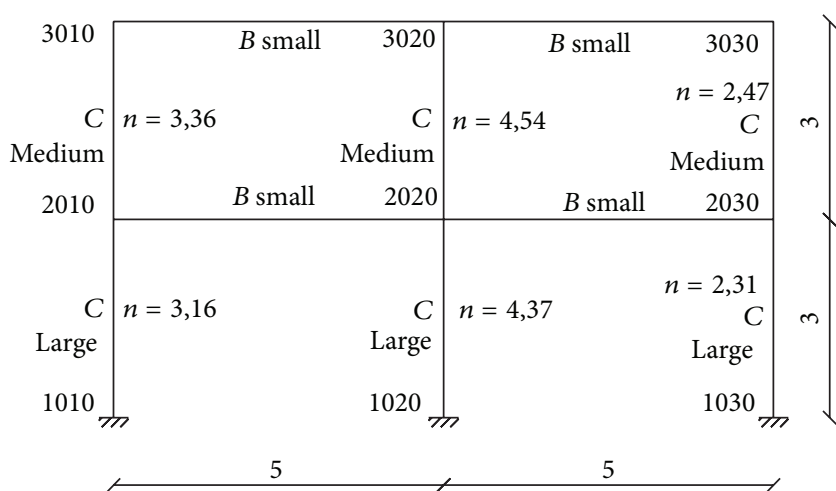

FIGURE 3: Hyperstatic structure.

reinforced concrete shown in Figure 3 subject to forms of degradation referable to the ULS [23].

The situation is more complex, because the collapse mechanism in hyperstatic structures is not unique and depends not only on the geometry but also on the size and disposition of loads [28-30]. Each structural element has its own weight in relation to the collapsing mechanism.

The expression of degradation will therefore depend on the relevance of the structural element in respect to the whole structure.

It becomes necessary to assign not only the weight that a single expression of degradation has on safety but also the weight that the structural element has on the structure in its entirety, whether it is the beam or the column. It is essential to assign a different resistance to the various structural elements in order to understand which element might collapse sooner than the others.

For this purpose a nonlinear elastic analysis on the twodimensional structure type (Figure 3 ) was performed with lumped plasticity in accordance with EN 1998-1:2004.

The purpose of this study is not to perform a test, but to identify, through the formation of plastic hinges, the sections that reach the yield point and form the collapse
TABLE 1: Structure geometry.

\begin{tabular}{lc}
\hline Length of the spans in the $x$ direction & $l_{x}=5 \mathrm{~m}$ \\
Length of the spans in the $y$ direction & $l_{y}=4.5 \mathrm{~m}$ \\
Floor height & $h=3 \mathrm{~m}$ \\
Slab thickness & $h_{f}=0.26 \mathrm{~m}$ \\
\hline
\end{tabular}

mechanism [28]. In fact, if the structure is hyperstatic, that is to say, equipped with external constraints larger in number than those statically sufficient to ensure balance, the formation of a plastic hinge generally does not produce labilization and for this purpose it is necessary that more hinges are activated or occur in different positions in order to allow a collapse mechanism.

The hypothesis of considering the two-dimensional structure is valid, because the building in the chosen example is regular.

In order to verify the method, a seismic action was applied to the structure. The seismic action is characterized by the accelerometric horizontal component of the seismic motion. For the calculations, the software DRAIN-2DX (DRAIN2DX: static and dynamic analysis of inelastic plane structures, software developed at the University of Illinois at UrbanaChampaign and provided by Professor Mark Aschheim; it is a finite element program for the seismic analysis of linear and nonlinear plane frames) was used. This software applies accelerograms of known seismic events such as the one relative to the earthquake which occurred in El Centro, Imperial Valley, California (USA), in 1940 whose diagram, as a function of time, will be shown later (Figure 5).

\subsection{Input Data. See Tables 1, 2, 3, and 4.}

4.2. Ductility Class. The structure is tested for medium ductility class. Rotations of the columns at foundation level are prevented (joint constraints). The horizontal elements are considered to be infinitely rigid; that is, it is assumed that there are no relative movements between the different points on the same plane. This hypothesis is valid given the nature of the slab, constructed in reinforced concrete.

4.3. Numbering of the Nodes. The numbers of the nodes inserted in the data input of the program are shown in Figure 3.

4.4. Loads and Masses. Types of loads applied to the structure are as follows:

(i) Permanent loads:

(1) $g_{s}$ : weight of the floor;

(2) $g_{s}=\gamma_{c} \cdot h_{f}=25 \cdot 0.26=6.5 \mathrm{KN} / \mathrm{m}^{2}$;

(3) $g_{c}$ : weight columns;

(4) $g_{b}:$ weight beams;

(5) $g_{f}=1 \mathrm{KN} / \mathrm{m}^{2}$ brought permanent coverage;

(6) $g_{f}=0.5 \mathrm{KN} / \mathrm{m}^{2}$ permanent brought intermediate floors. 
TABLE 2: Section geometry.

\begin{tabular}{|c|c|c|}
\hline \multirow{7}{*}{ Columns $c_{s}=C$} & $b_{c}$ column width in the $y$ direction & $30 \mathrm{~cm}$ \\
\hline & $h_{c}$ column height in the $x$ direction & $30 \mathrm{~cm}$ \\
\hline & $A_{c}$ area of cross sections & $900 \mathrm{~cm}^{2}$ \\
\hline & $I_{c y}$ moment of inertia about the $y$-axis & $67500 \mathrm{~cm}^{4}$ \\
\hline & $A_{c s}$ is the effective area to cut $A_{c s}=0.83 A_{c}$ & $747 \mathrm{~cm}^{2}$ \\
\hline & Steel reinforcement columns on the ground floor & $(3+3) \varphi 14$ \\
\hline & Steel reinforcement columns on the first floor & $(2+2) \varphi 14$ \\
\hline \multirow{10}{*}{ Beams $c_{s}=B$} & $b_{w}$ width of the web in the $y$ direction & $30 \mathrm{~cm}$ \\
\hline & $h_{w}$ is the height of the floor in the $x$ direction & $50 \mathrm{~cm}$ \\
\hline & $b_{f}$ flange width in the $y$ direction & $186 \mathrm{~cm}$ \\
\hline & $h_{f}$ height of the flange in the $x$ direction & $26 \mathrm{~cm}$ \\
\hline & $A_{b}$ cross section area & $6336 \mathrm{~cm}^{2}$ \\
\hline & $z_{g}$ distance of center of gravity from the upper edge & $22 \mathrm{~cm}$ \\
\hline & $I_{b y}$ moment of inertia around the $y$-axis & $2238144 \mathrm{~cm}^{4}$ \\
\hline & $A_{b s}$ is the effective area to cut $A_{b s}=b_{w}\left(h_{w}+h_{f}\right)$ & $1680 \mathrm{~cm}^{2}$ \\
\hline & Steel reinforcement in the tension zone & $4 \varphi 14$ \\
\hline & Steel reinforcement in the compression zone & $3 \varphi 14$ \\
\hline
\end{tabular}

TABLE 3: Variable loads.

\begin{tabular}{|c|c|c|}
\hline $\begin{array}{l}\text { Intermediate floors: } \\
\text { Reference Category C1 } \\
\text { (subject to crowding) }\end{array}$ & \multicolumn{2}{|c|}{$q_{k}=3 \mathrm{KN} / \mathrm{m}^{2}$} \\
\hline $\begin{array}{l}\text { Top floor: } \\
\text { Reference Category H } \\
\text { (H1 accessible for maintenance only) }\end{array}$ & \multicolumn{2}{|c|}{$q_{k}=0.5 \mathrm{KN} / \mathrm{m}^{2}$} \\
\hline \multicolumn{3}{|c|}{ TABLE 4: Values loads. } \\
\hline Floors & Cover & First floor \\
\hline \multicolumn{3}{|c|}{ Distributed loads $(\mathrm{KN} / \mathrm{m})$} \\
\hline Dead weight floor & 29.25 & 29.25 \\
\hline Dead weight beam in the $x$ direction & 3.75 & 3.75 \\
\hline Variables & 1.35 & 6.48 \\
\hline Total load & 34.35 & 39.48 \\
\hline \multicolumn{3}{|c|}{ Point loads (KN) exterior columns } \\
\hline Dead weight beam in the $y$ direction & 16.87 & 16.87 \\
\hline Upper column & 0 & 3.37 \\
\hline Lower column & 3.37 & 3.37 \\
\hline$P_{e, \text { tot }}$ & 20.25 & 23.61 \\
\hline \multicolumn{3}{|c|}{ Point loads $(\mathrm{KN})$ interior columns } \\
\hline Dead weight beam in the $y$ direction & 16.87 & 16.87 \\
\hline Upper column & 0 & 3.37 \\
\hline Lower column & 3.37 & 3.37 \\
\hline$P_{i, \text { tot }}$ & 20.25 & 23.61 \\
\hline
\end{tabular}

(ii) Variable loads:

(1) $q_{k}=0.5 \mathrm{KN} / \mathrm{m}^{2}$ variable coverage;

(2) $q_{k}=3 \mathrm{KN} / \mathrm{m}^{2}$ variables intermediate floors.
4.5. Load Combinations. In order to determine the nodal masses, the following load combination was used:

$$
\sum G_{k j} \cup \sum \Phi_{E i} Q_{k i}
$$

where

(i) $G_{k j}$ and $Q_{k i}$ are, respectively, the characteristic values of permanent actions and variables;

(ii) $\Phi$ is equal to 1 for the coverage and 0.8 for intermediate floors; $\Psi_{E i}$ is a combination coefficient for the $i$ th variable action; these coefficients take into account the probability that the loads $Q_{k i}$ do not act simultaneously on the entire structure during the earthquake; it assumes the value of 0.6 for the variable actions in a crowding and the value of 0 for the variable action due to wind or snow.

For each floor, the following load distribution will be considered:

(i) Distributed loads:

(1) weight of the floor: $1.0 \cdot g_{s} \cdot l_{y}=1.0 \cdot 6.5 \cdot 4.5=$ $29.25 \mathrm{KN} / \mathrm{m}$;

(2) weight of the beam in the $x$ direction: $\gamma_{c} \cdot b_{w} \cdot h_{w}=$ $1.0 \cdot 25 \cdot 0.3 \cdot 0.5=3.75 \mathrm{KN} / \mathrm{m}$;

(3) variable load coverage: $1.0 \cdot \varphi \cdot 0.6 \cdot l_{y} \cdot q_{k}=$ $1.0 \cdot 1 \cdot 0.6 \cdot 0.5 \cdot 4.5=1.35 \mathrm{KN} / \mathrm{m}$

(4) variable load intermediate floors: $1.0 \cdot \varphi \cdot 0.6 \cdot l_{y}$. $q_{k}=1.0 \cdot 3 \cdot 0.8 \cdot 0.6 \cdot 4.5=6.48 \mathrm{KN} / \mathrm{m}$.

(ii) Point loads:

(1) weight of the beam in the $y$ direction: $1.0 \cdot \gamma_{c}$. $l_{y} \cdot b_{w} \cdot h_{w}=3.75 \cdot 4.5=16.87 \mathrm{KN}$; 
TABLE 5: Vertical loads.

\begin{tabular}{lcccccccc}
\hline \multirow{2}{*}{ Floors } & \multicolumn{4}{c}{ External nodes } & \multicolumn{3}{c}{ Internal nodes } \\
& $P_{e}(\mathrm{KN})$ & $R_{e}(\mathrm{KN})$ & $P_{e}+R_{e}(\mathrm{KN})$ & $M_{e}(\mathrm{KNm})$ & $P_{i}(\mathrm{KN})$ & $R_{i}(\mathrm{KN})$ & $P_{i}+R_{i}(\mathrm{KN})$ & $M_{i}(\mathrm{KNm})$ \\
\hline Cover & 20.25 & 85.87 & 106.12 & 71.56 & 20.25 & 171.75 & 192 & 0 \\
First floor & 23.62 & 98.70 & 122.32 & 82.25 & 23.62 & 197.4 & 221.02 \\
\hline
\end{tabular}

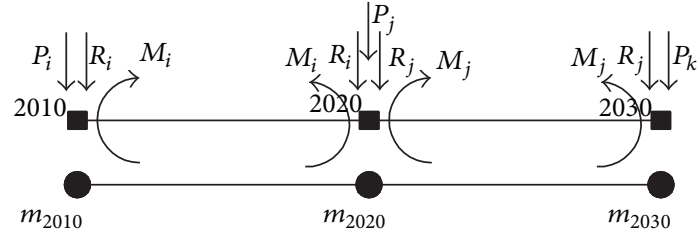

Figure 4: Nodal loads.

(2) weight of the upper column: $1.0 \cdot \gamma_{c} \cdot b_{c \text {, sup }} \cdot h_{c \text {, sup }} \cdot$ $h / 2$;

(3) weight of the lower column: $1.0 \cdot \gamma_{c} \cdot b_{c \text {, inf }} \cdot h_{c \text {, inf }}$. $h / 2$.

The values are given in Table 4.

4.6. Nodal Loads. It is assumed that the beams are fixed at their ends. Therefore the vertical reactions at the supports and the moments are equal to

$$
\begin{gathered}
R_{i}=\frac{q_{\mathrm{tot}} \cdot l_{x}}{2} ; \\
M_{i}=\frac{q_{\mathrm{tot}} \cdot l_{x}^{2}}{12} .
\end{gathered}
$$

Horizontal loads in the nodes are zero, while the vertical loads are given by the sum of the reactions $R_{i}$ and $P_{i}$ point loads indicated in Table 4. Regarding the $M_{i}$ moments at the nodes, they must be added (with their signs) in the internal nodes. A scheme for the nodal loads with respect to the first floor is shown in Figure 4.

Nodal load values for each floor are shown in Table 5, where the nodes are divided into internal and external.

4.7. Nodal Masses. Nodal masses are obtained by dividing the vertical loads concentrated at each node by the acceleration of gravity $\left(g=9.81 \mathrm{~m} / \mathrm{sec}^{2}\right)$. So, for example, for the nodes of the first floor (Figure 4) we have

$$
\begin{aligned}
& m_{2010}=\frac{P_{i}+R_{i}}{g} ; \\
& m_{2020}=\frac{P_{j}+R_{i}+R_{j}}{g} ; \\
& m_{2030}=\frac{P_{j}+R_{j}}{g} .
\end{aligned}
$$

Based on the convection of signs adopted by the software DRAINDX forces pointing downwards are negative, while counterclockwise moments are positive.

The values of the nodal masses are given in Table 6 .
TABLE 6: Nodal masses.

\begin{tabular}{lcc}
\hline Floors & Node & $\begin{array}{c}\text { Nodal masses } \\
m\left(\mathrm{KN} /\left(\mathrm{cm} / \mathrm{sec}^{2}\right)\right)\end{array}$ \\
\hline Cover & 3010 & 0.108 \\
& 3020 & 0.196 \\
& 3030 & 0.108 \\
\hline \multirow{3}{*}{ First floor } & 2010 & 0.125 \\
& 2020 & 0.225 \\
& 2030 & 0.125 \\
\hline
\end{tabular}

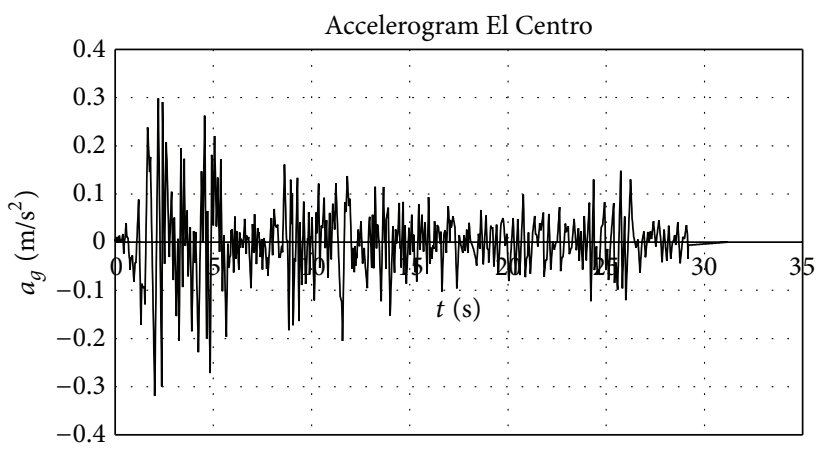

Figure 5: Accelerogram El Centro, Imperial Valley, California, in 1940.

\section{Analysis of the Structure with Lumped Plasticity}

The results obtained are shown below by applying the accelerogram corresponding to the earthquake which occurred in El Centro, Imperial Valley, California, in 1940 (Figure 5).

The history of the development of plastic hinges in the structure under examination is shown in Figure 6, where the filled circles represent the plastic hinges formed at time $t$, while the empty circles symbolize the plastic hinges developed earlier. The first plastic hinge takes place in the most stressed section, and, therefore, any further increase of load causes other plastic hinges until the structure becomes a mechanism.

This nonsimultaneous formation of plastic hinges involves significant plastic rotations in the hinges that were formed first, which allow the redistribution of moments between critical sections [28].

It can be noted in Figure 6 that at $t=1.3210 \mathrm{sec}$ (earthquake in El Centro) in the structure a soft floor 


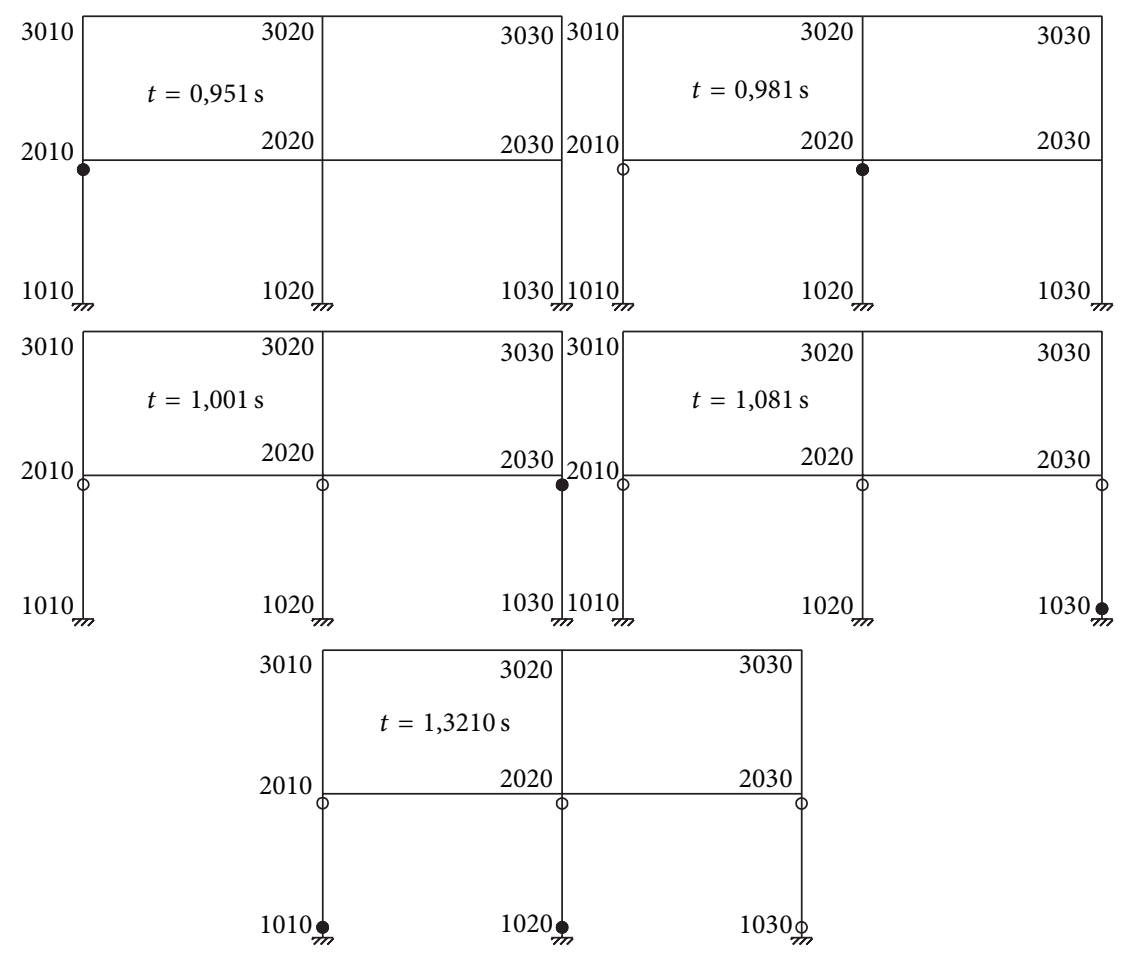

FIgURE 6: Development of plastic hinges.

mechanism has taken place that involves yielding on a large part of the columns of the ground floor, before occurring in the beams. A global fragile mechanism was activated.

It is therefore clear how a possible reduction of the section of the lower columns, caused by any type of damage, will further weaken the structure and anticipate collapse.

The weight of columns of the ground floor is greater than the weight of the other elements of the structure. We could therefore build a hierarchy of elements following the story of formation of the plastic hinges. The problem is that this type of analysis can be performed only when the construction under inspection is perfectly known in terms of the geometry of the structure and sections and also of the applied loads.

At this point, it is necessary to give a priori weights to the different elements of the structure according to the regulations $[29,30]$, which constructions have to observe in order that the more fragile parts of the constructions are, for example, more protected against failure mechanisms.

In this way, the weight that each structural element has in a hyperstatic structure may coincide with the optimal mechanism of development of plasticization.

Thinking in terms of weight, a greater weight to the columns should be assigned in respect to the beams and, within this differentiation, it is necessary to give greater weight to events related to shear degradation rather than to those related to bending. The remaining problem is therefore to assign to the columns a greater weight than to the beams, also taking into consideration that the columns of the ground floor must have greater weight than those of the first floor.

\section{Fuzzy Logic Applied to Visual Inspection}

The aim of this study is to manipulate through fuzzy logic the subjective linguistic judgments expressed by an inspection staff on the visual signs of degradation in order to assess the current safety level of the inspected construction [12-15, 21].

The method is consequently divided into two stages: the first consists in the insertion of data obtained by visual inspection on a standard form card (Inspection Card), eventually attaching to it photographs and videos [31]; the second one, carried out by expert staff, involves the implementation of the analysis method by means of software developed by the authors [12-15] and further extended for the case under analysis. The data, collected in the Inspection Card, is included in the Evaluation Card shown in Table 7.

The card consists of five columns. In the first column the structural element under inspection is indicated.

In the second column for each structural element the degradation expressions that are more relevant to the structural element itself are shown. In the third column the inspection staff will write down the assessments of gravity attributed to the different types of degradation. The fourth column shows the weights that each lexical judgment will have on the current safety assessments. The fifth indicates the weight that the structural element holds within the structure under consideration. It is important to stress that the card and the relationship between judgment and weight are constructed according to the expertise of a technically skilled team, while the third column is filled in during the visual inspection by the staff. 
TABLE 7: Evaluation Card.

\begin{tabular}{lcccc}
\hline Structural element & Type of degradation & Linguistic judgment & Safety goal & Weight of the structural element \\
\hline$E_{i}$ & $E_{i j}$ & $G_{i j k}$ & $W_{i j k l}$ & $W E_{i j k l m}$ \\
\hline
\end{tabular}

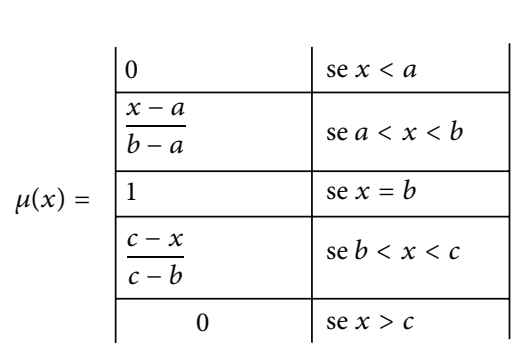

(a)

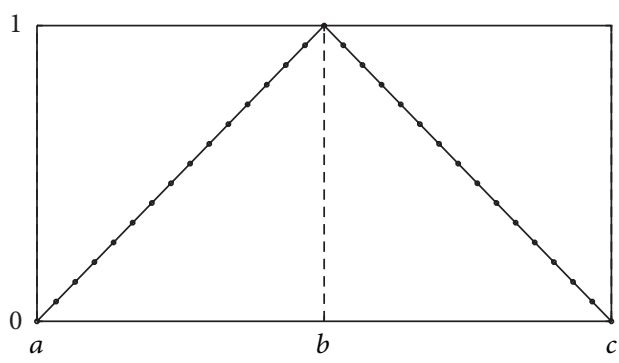

(b)

Figure 7: Developments of the triangular membership functions.

Our research proposes a manipulation of linguistic subjective judgments expressed by the inspection staff on the degradation of a structure using fuzzy logic. The goal is to assess the current safety level of the inspected construction.

The operator gives a linguistic judgment for each type of degradation $E_{i j}$. Thus, we have the crisp set of linguistic judgments: $G_{i j k}$ (small (S), medium (M), and large (L)), between which the inspector chooses the most suitable to represent the level of degradation, which is a subjective choice.

With each linguistic judgment $G_{i j k}$ the weight $W_{i j k l}$ that it has on the current safety level using a crisp set of linguistic judgments is associated: $W_{i j k l}$ (very small (VS), small (S), slightly small (SLS), medium (M), slightly large (SLL), large $(\mathrm{L})$, and very large $(\mathrm{VL})$ ). The weight is defined following the considerations of the expert staff.

For each structural element $E_{i}$, the weight $W E_{i j k l m}$ that the element has within the structure is given by the skilled staff, using a set of crisp linguistic judgments: $W E_{i j k l m}$ (small $(\mathrm{S})$, medium $(\mathrm{M})$, and large $(\mathrm{L})$ ).

The linguistic variables represent our quality input (Figure 1).

With each linguistic variable we associate a membership function $\mu(x)$ whose domain $X$ is $[0,1]$. This function associates with each value $x$ belonging to the range $X[0,1]$ a number $\mu(x)$ in the range $[0,1]$ that represents the degree of membership of $x$ in $X$; 1 represents the maximum degree of membership and 0 the minimum degree. This interval will be discretized in order to obtain the vectors representative of the individual fuzzy functions, to which the fuzzification, inference, and defuzzification procedures will be applied.

For both the judgments $G_{i j k}$ and the weights $W_{i j k l}$, the triangular membership functions shown in Figures 7 and 8 were chosen. It should be noted that identical functions $\mathrm{S}, \mathrm{M}$, and $\mathrm{L}$ for both the judgment $G_{i j k}$ and the weight $W_{i j k l}$ have been chosen, but this is not mandatory.

The membership functions $\mu(x)$, associated with each linguistic variable small, medium, and large of the weight $W E_{i j k l m}$, have a domain, $X$, between 0 and 1 . For the judgments $G_{i j k}$, for the weights $W_{i j k l}$, and for the weight

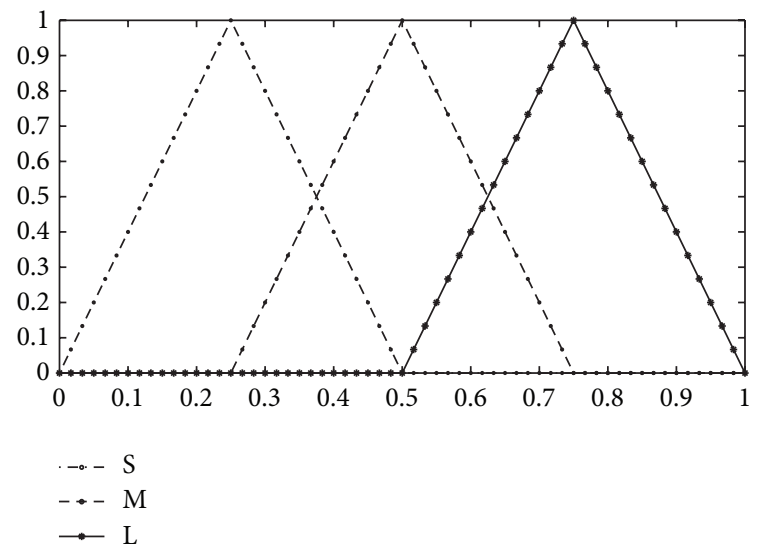

FIGURE 8: The membership functions of linguistic judgments: $G_{i j k}$ ( small (S), medium (M), and large (L)).

$W E_{i j k l m}$ the triangular membership functions were chosen as shown in Figure 7. For the judgement $G_{i j k}$, for the weight $W_{i j k l}$, and for the weight $W E_{i j k l m}$ the fuzzy functions domain $[0,1]$ was discretized in $N_{f}=41$ points with 40 intervals equal to 0.025 (Figures 8, 9, and 10) thus obtaining a $41 \times 1$ vector.

In Table 8 and in Table 9 the values for $a, b$, and $c$ for the chosen membership functions for $G_{i j k}, W_{i j k l}$, and $W E_{i j k l m}$ are shown.

The variation range of the domain of the weight $W E_{i j k l m}$ varies from -1 to +0.25 , the origin is coincident with safety exponent $n$ defuzzificated. This discretization proved to be fine enough for the authors' method. Using smaller intervals did not improve results. After several attempts of optimization, it was found that the choice is strictly related to the type of application. It was decided to use the triangular asymmetrical membership functions shown in Figure 10 in which the value of the maximum membership function small corresponds to the average value of the safety exponent $n$ defuzzificated. 
TABle 8: Values $a, b$, and $c$ of membership functions $G_{i j k}, W_{i j k l}$, and $N_{e}$.

\begin{tabular}{|c|c|c|c|c|c|c|c|}
\hline $\begin{array}{l}\text { Membership functions } \\
G_{i j k} \text { and } W_{i j k l}\end{array}$ & $a$ & $b$ & $c$ & $\begin{array}{c}\text { Membership functions } \\
\qquad N_{e}\end{array}$ & $a$ & $b$ & $c$ \\
\hline VS & - & 0 & 0.1 & VSS & 7 & 6.5 & - \\
\hline S & 0 & 0.25 & 0.5 & SS & 7 & 5.75 & 4.5 \\
\hline SLS & 0.25 & 0.4 & 0.5 & SLSS & 5.75 & 5 & 4.5 \\
\hline M & 0.25 & 0.5 & 0.75 & MS & 5.75 & 4.5 & 3.25 \\
\hline SLL & 0.5 & 0.6 & 0.75 & SLLS & 4.5 & 4 & 3.25 \\
\hline $\mathrm{L}$ & 0.5 & 0.75 & 1 & LS & 4.5 & 3.25 & 2 \\
\hline VL & 0.9 & 1 & - & VLS & - & 2.25 & 2 \\
\hline
\end{tabular}

TABLE 9: Values $a, b$, and $c$ of membership functions $W E_{i j k l m}$.

\begin{tabular}{lccc}
\hline Membership functions & $a$ & $b$ & $c$ \\
$W E_{i j k l m}$ & & $n$ & 0.25 \\
$\mathrm{~S}$ & -0.25 & -0.5 & $n$ \\
$\mathrm{M}$ & -0.75 & -0.75 & $n$ \\
$\mathrm{~L}$ & -1 & \\
\hline
\end{tabular}

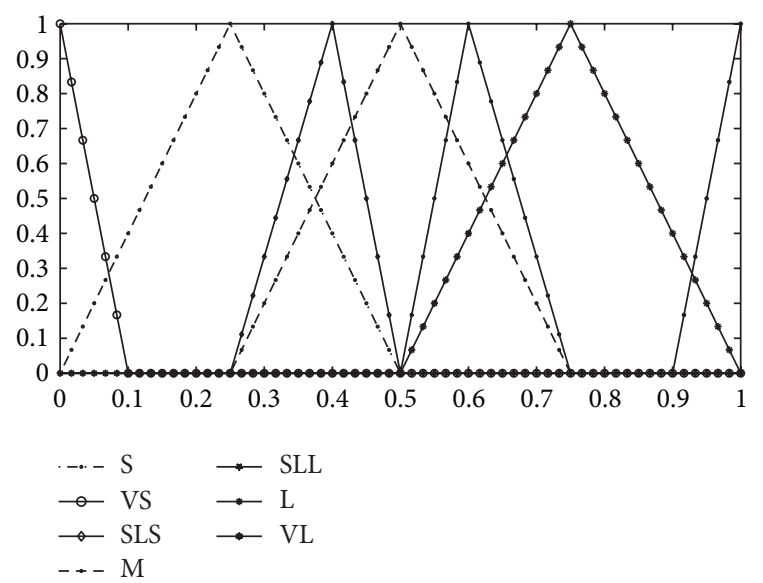

FIGURE 9: The membership functions of weight: $W_{i j k l}$ (very small (VS), small (S), slightly small (SLS), medium (M), slightly large (SLL), large (L), and very large (VL)).

\section{Application of the Proposed Method}

The proposed method was applied to the generic hyperstatic structure shown in Figure 3, subject to forms of degradation of the type ULS, giving to the structural elements the weight that they have inside the building in question. It is important to stress that this weight is defined by experts before the inspection takes place. Based on the above considerations, the weight large was assigned to the pillars that constitute the ground floor, medium to those constituting the first floor, and finally small to the beams (Figure 3 ).

It is assumed that the structure in question is in an advanced state of degradation and that the Evaluation Card is the one shown in Table 10 for the beams and in Table 11 for the columns.

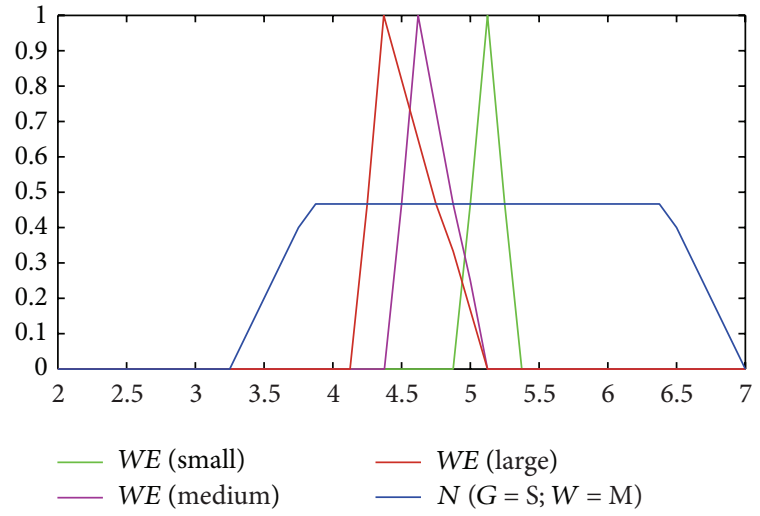

Figure 10: The membership functions of the weight that the structural element has within the structure: $W E_{i j k l m}$ (small (S), medium (M), and large (L)).

In the last column the safety factors obtained by varying the $n$ possible linguistic judgments (small, medium, and large) are listed. An example is shown where an exponent safety $n$ is obtained equal to 5.12 using the proposed procedure. This value is obtained by giving the judgment small to the event of degradation. The weight of such a safety issue is medium.

The procedures suggested in the literature are two. The first, based on fuzzy sets theory [8-10], follows the approach suggested by Blockley $[6,7]$ using the intersection operator, merge and fuzzy relations. The second procedure, recommended by Tee et al. [32], is based on the algebra of fuzzy numbers [8-10] with the use of the weighted average. In this study we followed and extended the first procedure in accordance with Acito [21].

The intersection operator initially combines the single judgment $G_{i j k}$ with the weight $W_{i j k l}$ that it has on structural safety expressed vectorially in the following form:

$$
C \widetilde{D}_{i}=\widetilde{G}_{i j} \cap \widetilde{W}_{i j k l} .
$$

Given two fuzzy sets, for example, $G_{i j k}=$ small and $W_{i j k l}=$ medium, the intersection between small and medium is defined as the fuzzy set $D=S \cap M$ having the characteristic function shown in Figure 11:

$$
\mu_{D(x)}=\min \left(\mu_{s}(x), \mu_{M}(x)\right) ; \quad \forall x \in X .
$$


TABLE 10: Degradation assessment card beams.

\begin{tabular}{lcccc}
\hline Structural element $E_{i}$ & Type of degradation $E_{i j}$ & Linguistic judgement $G_{i j k}$ & Safety goal $W_{i j k l}$ & Safety exponent $n$ \\
\hline & & Small & Medium & 5.12 \\
& Shear stress & Medium & Large & 3.88 \\
Beam & & Large & Very large & 3.00 \\
& Longitudinal stress & Small & Slightly small & 5.62 \\
& & Medium & Medium & 4.50 \\
& Large & Large & 3.25 \\
\hline
\end{tabular}

TABLE 11: Degradation assessment card columns.

\begin{tabular}{lcccc}
\hline Structural element $E_{i}$ & Type of degradation $E_{i j}$ & Linguistic judgement $G_{i j k}$ & Safety goal $W_{i j k l}$ & Safety exponent $n$ \\
\hline & & Small & Medium & 5.12 \\
& Reinforcement corrosion & Medium & Large & 3.88 \\
Column & & Large & Very large & 3.00 \\
& Spalling & Small & Slightly small & 5.62 \\
& & Medium & Medium & 4.50 \\
& & Large & Large & 3.25 \\
\hline
\end{tabular}

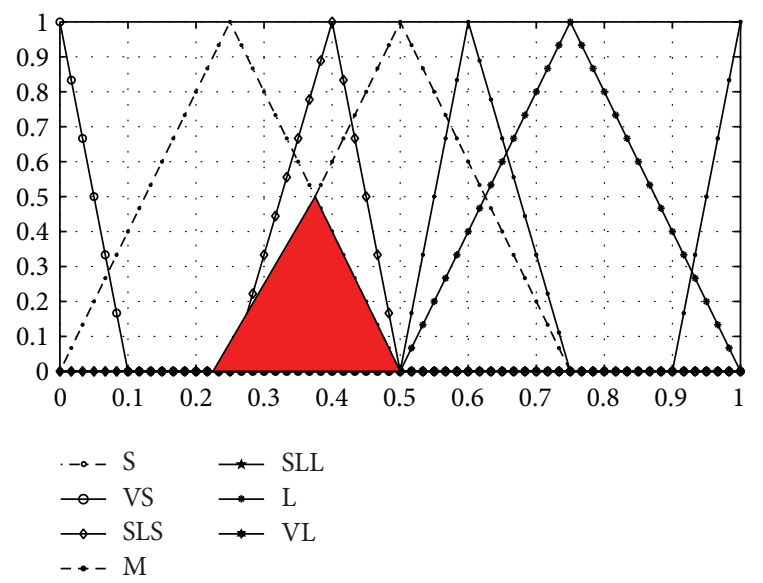

FIGURE 11: The intersection of two fuzzy sets $G_{i j k}=$ small and $W_{i j k l}=$ medium; graphic of the characteristic function intersection.

The fuzzy functions are represented by vectors $1 \times N_{f}$, and a discretization was chosen such that $N_{f}=41$. Therefore, the result of the procedure of intersection is a vector $1 \times 41$.

The combined effect of the judgment and the weight relative to each degradation was calculated (6), in order to evaluate whether the security level of an a priori assumption for the building visually inspected was altered. The security level is expressed by the initial value of the safety exponent $n$. Following the regulations $[22,23]$, it can be seen that $n$ has values ranging from 1 to 7 , according to the limit state.

In the proposed method the variation range of the exponent is assumed to be the domain of the security level. Since the safety measurement is positive if $n \geq 4$, the value 7 represents the optimum value.

It is assumed a priori that the hypothesis of the structure under examination is designed and constructed in conformity with the regulations.
In this case we assume the structure, in presence of the loads required by regulations, to be in a safe condition. Therefore, if we refer to the value of the safety measure usually denoted by $P_{f}=10^{-n}$ [21] we can assume an a priori value of the exponent $n$ equal to 7 .

We need then to establish a fuzzy relation between the judgment of a single type of deterioration and the evaluation of the safety exponent, by adopting a fuzzy composition according to

$$
\widetilde{R}(\widetilde{G} \times \widetilde{N})=\widetilde{R}(\widetilde{G} \cap \widetilde{W}) \circ \widetilde{R}(\widetilde{W} \times \widetilde{N}) .
$$

We know the value of the fuzzy relation $R(G \cap W)$ given by (6), while in order to derive the fuzzy relation $R(W \times N)$ we need to use the inference procedure based on logical operations. We thus have the following rules of inference [8$10,21]$ :

If $W_{i j k l}$ is very small then $P_{f}$ is very small and $n$ is very large.

If $W_{i j k l}$ is small then $P_{f}$ is small and $n$ is large.

If $W_{i j k l}$ is slightly small then $P_{f}$ is slightly small and $n$ is slightly large.

If $W_{i j k l}$ is medium then $P_{f}$ is medium and $n$ is medium.

If $W_{i j k l}$ is slightly large then $P_{f}$ is slightly large and $n$ is slightly small.

If $W_{i j k l}$ is large then $P_{f}$ is large and $n$ is small.

If $W_{i j k l}$ is very large then $P_{f}$ is very large and $n$ is very small.

With each linguistic variable used for the safety exponent $n$, membership function $\mu(x)$ is associated, and a triangular shape was chosen, between $n_{\max }=7$ and $n_{\min }=2$, as shown by Figure 12. The fuzzy functions corresponding to the safety exponent $n$ are represented by vectors $1 \times N_{e}$. For the safety 


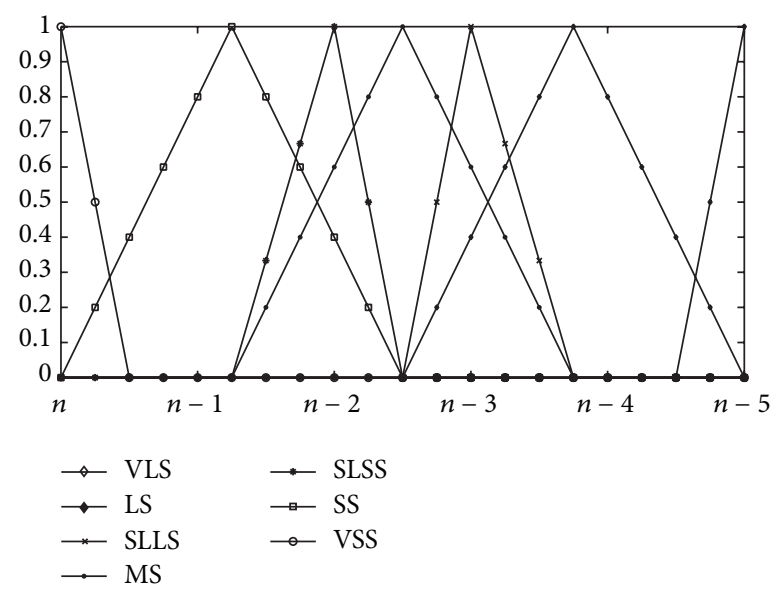

FIGURE 12: The membership functions of safety exponent: $N_{e}$ (very small (VSS), small (SS), slightly small (SLSS), medium (MS), slightly large (SLLS), large (LS), and very large (VLS)).

exponent $N_{e}$ we discretize the domain $[2,7]$ of the fuzzy function using $N_{e}=21$ points and therefore 20 intervals of width equal to 0.05 , thus obtaining a vector of dimensions $21 \times 1$. In Table 8 we showed the values for $a, b$, and $c$ for the chosen $N_{e}$ membership functions.

$N=$ (very small (VSS), small (SS), slightly small (SLSS), medium (MS), slightly large (SLLS), large (LS), and very large (VLS)). in (9).

The fuzzy relation $R(W \times N)$ can be written as specified

A fuzzy relation is a fuzzy set defined on multiple domains (multidimensional fuzzy set). Once expressed the fuzzy relations $R_{i}(i=1, \ldots, 7)$, with the merge operation between the fuzzy relations $R_{i}$ in $X \times Y$, were proceeded. The union operation is defined in (10).

Figure 13 shows graphically the result of the operation. A matrix of size $N_{f} \times N_{e}$ was obtained, in this case $41 \times 21$ :

$$
\begin{aligned}
\widetilde{R}(W \times N)= & \widetilde{R}_{1}(\widetilde{W} \times \widetilde{N}) \cup \widetilde{R}_{2}(\widetilde{W} \times \widetilde{N}) \\
& \cup \widetilde{R}_{3}(\widetilde{W} \times \widetilde{N}) \cup \widetilde{R}_{4}(\widetilde{W} \times \widetilde{N}) \\
& \cup \widetilde{R}_{5}(\widetilde{W} \times \widetilde{N}) \\
& \cup \widetilde{R}_{6}(\widetilde{W} \times \widetilde{N}) \cup \widetilde{R}_{7}(\widetilde{W} \times \widetilde{N}), \\
R_{1} \cup R_{2}= & \left\{\left((x, y), \mu_{R 1 \cup R 2}(x, y)\right) \mid \mu_{R 1 \cup R 2}(x, y)\right\} \\
= & \max \left\{\mu_{R 1}(x, y), \mu_{R 2}(x, y)\right\}
\end{aligned}
$$

with $(x, y)$ belonging to $X \times Y$ :

$$
\mu_{\widetilde{R}(\widetilde{G} \times \widetilde{N})}=\sup \left(\min \left\{\mu_{\widetilde{R}(\widetilde{G} \cap \widetilde{W})}, \mu_{\widetilde{R}_{(\widetilde{W} \times \widetilde{N})}}\right\}\right) .
$$

The operation of combination (8) combines the fuzzy relations in different product spaces. In this study, reference was made to the composition of fuzzy relations proposed by Zadeh [4].

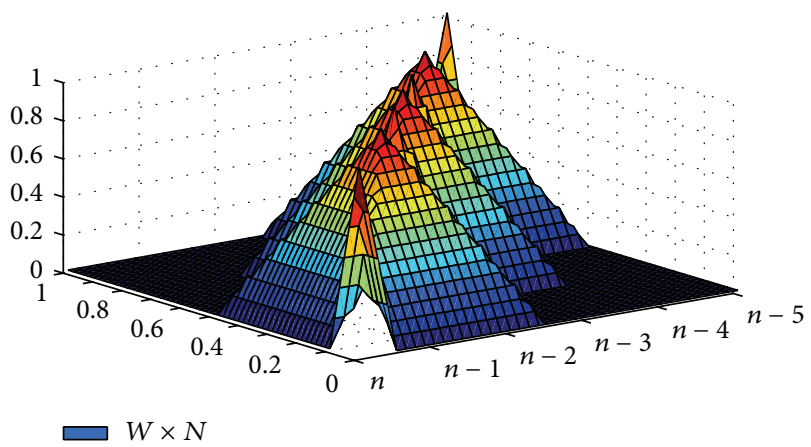

Figure 13: $W \times N$ plot in space.

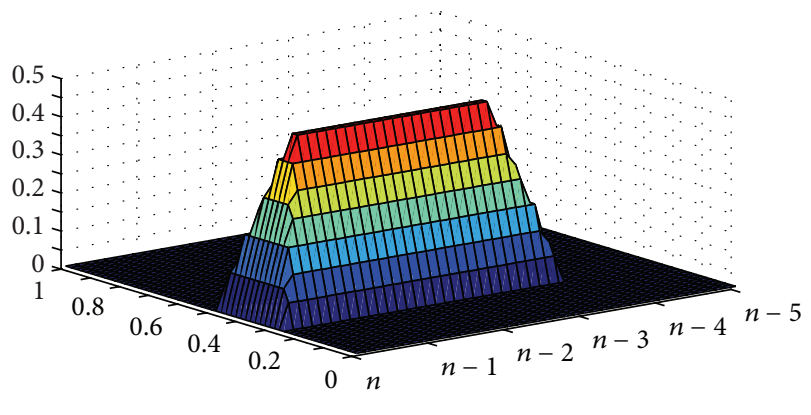

$G \times N G=S ; W=M$

FIgURE 14: $G \times N$ plot in space.

The result of this composition (Figure 14) is a fuzzy set whose membership function is shown in (11).

The result of the inference procedure gives the relation between the weight on the safety and the safety exponent itself; the result is expressed by a matrix $N_{e} \times 1$ which is obtained by considering the maximum value of each column of the final matrix $(\widetilde{G} \times \widetilde{N})$ :

$$
n_{j}=\max \left(\mu_{\widetilde{R}(\widetilde{G} \times \widetilde{N})}\right)_{j, i \in[0,1]} .
$$

In Figure 15 the value of the fuzzified safety factor was plotted giving visual lexical judgment $G_{i j k}=$ small and weight $W_{i j k l}=$ medium, and this result is expressed by a onedimensional array $41 \times 1$.

In order to evaluate the effect that the weight has on the value of the safety exponent it is necessary to assess its value by defuzzifying the result (Figure 15) obtained in terms of a fuzzy set. It is necessary to get the value of the exponent of security $n$ as a real number to be able then to compare it with the value of the same a priori assumption depending on the state reference limit (1).

In the literature [21] defuzzification is obtained by extracting the minimum value of the exponent $n$ which corresponds to the maximum membership. In this case, however, the results are not satisfactory because the defuzzified value does not vary in accordance with lexical judgement and weight variations. In the specific literature concerning fuzzy logic $[4,6-11]$ this value can be obtained in various ways as the method of the center of the area and the method of the average of the maximum. In this particular case, it was 


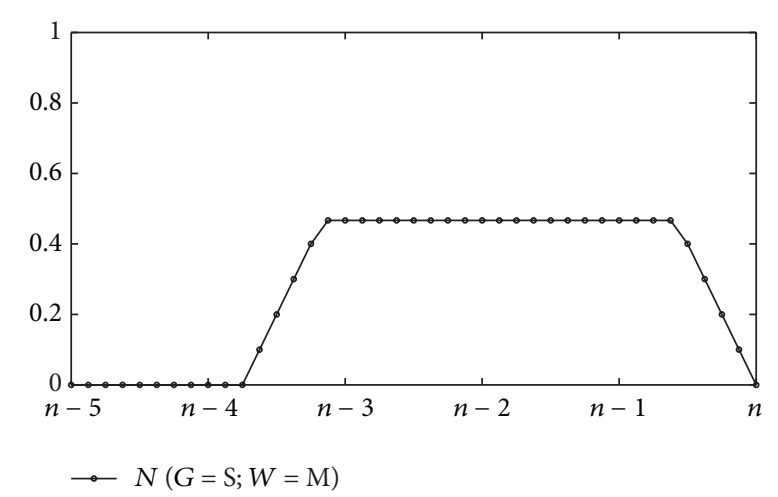

FIGURE 15: Safety exponent $G_{i j k}=\operatorname{small}(\mathrm{S}), W_{i j k l}=\operatorname{medium}(\mathrm{M})$.

considered convenient to take $n$ as the average value between the maximum value and the minimum value among those with maximum membership.

Giving the values $G_{i j k}=$ small and $W_{i j k l}=$ medium we obtain $n=5.12$, with a maximum value of 6.25 and a minimum value of 4.00 . It will be shown that the weight of the structural element $W E_{i j k l m}$ can change this value that so far was affected only by the weight of the event of degradation $W_{i j k l}$. The membership functions $\mu(x)$, associated with each linguistic variable small, medium, and large of the weight $W E_{i j k l m}$, have a domain membership of $X$ that is between 0 and 1 . We decided to use membership functions of triangular asymmetrical shape shown in Figure 10 in which the value corresponding to the maximum membership function small corresponds to the average value of the $n$ defuzzified safety exponent. As can be seen from Figure 10 the midpoint of the safety factor $n$ is equal to 5.12, associated with the judgment of gravity $G=$ small and weight $W=$ medium, which corresponds to the maximum value of the membership function small of the weight $W E$ associated with each structural element.

The value of the defuzzified exponent obtained from the final fuzzification procedure of Cartesian product $(N \times W E)$ is defuzzified by choosing the average of the values of $n$ with maximum membership. Tables 12 and 13 show the coefficients obtained by varying the final weight of the structural element.

In Figure 16 it can be seen that, giving the weight small to the structural element, this does not change the starting value of the safety exponent that is equal to 5.12. However, both the weights medium and large modify the value of the exponent, decreasing its value within the range defined by the experts. Giving medium weight a final value of the safety factor equal to 4.69 was obtained (Figure 17), while giving weight large an even smaller value, that is, 4.5 , was obtained (Figure 18).

In Tables 13 and 14 the linguistic values of gravity and the resulting value $n$ assigned to each expression of degradation are shown.

In the last column of the table there is only one safety factor that takes into account the presence of the two expressions.

To obtain a single value an operation similar to what is done to assess the probability of failure in the case of

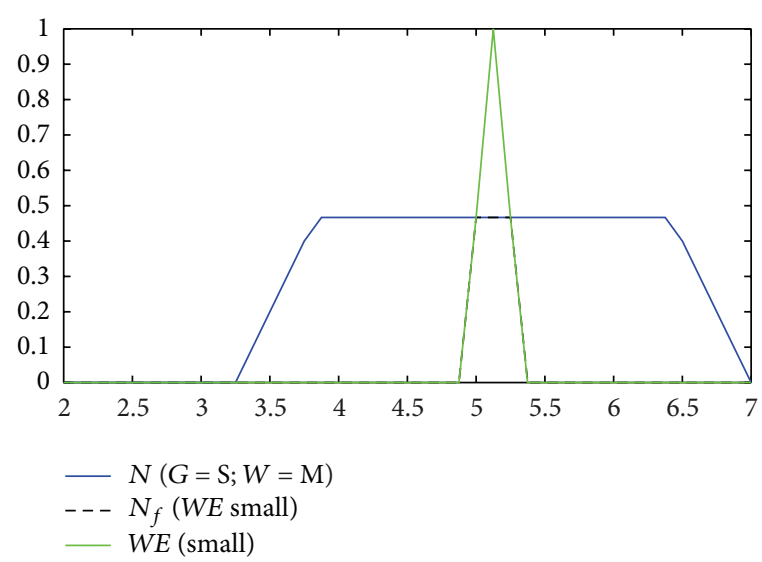

FIGURE 16: Safety exponent achieved by giving $G_{i j k}=$ small, $W_{i j k l}=$ medium, and $W E_{i j k l m}=$ small.

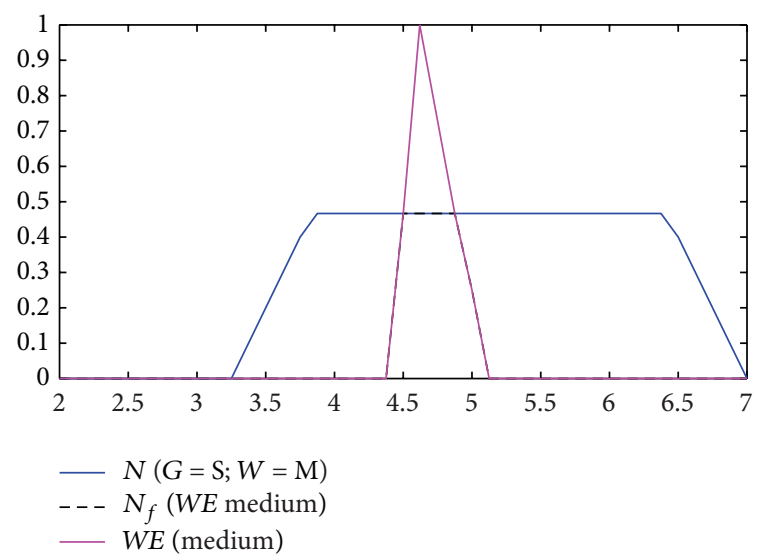

FIGURE 17: Safety exponent achieved by giving $G_{i j k}=$ small, $W_{i j k l}=$ medium, and $W E_{i j k l m}=$ medium.

a structural project was decided upon for which we have more causes of failure.

A chain mechanism is assumed, in series, in which the probability of failure of each event of degradation is identified with the probability of failure of a single link in the chain, and the probability of failure of the entire chain, in the hypothesis of independence of the results of each loop, takes the expression [12-15, 21, 33, 34]

$$
P_{f}=1-\prod_{i=1}^{m}\left(1-P_{f_{i}}\right)
$$

where $m$ is the number of degradation types. From this the value of the combined safety exponent $n$ is obtained.

\section{Analysis of Results}

In Figure 3, where each structural element is associated with a safety factor, it can be seen that although the same linguistic judgment of gravity has deliberately been given to the degradation expressions associated with the columns corresponding to different floors, the different weight given 
TABLE 12: Results columns.

\begin{tabular}{|c|c|c|c|c|c|c|c|}
\hline$E_{i}$ & $E_{i j}$ & $G_{i j k}$ & $W_{i j k l}$ & $n$ & $W E_{i j k l m}$ & $n$ & $n$ final \\
\hline \multirow{2}{*}{$E_{1010-2010}$} & $E_{11}$ reinforcement corrosion & Medium & Large & 3.88 & \multirow{2}{*}{ Large } & 3.25 & \multirow{2}{*}{3.16} \\
\hline & $E_{12}$ spalling & Medium & Medium & 4.50 & & 3.88 & \\
\hline \multirow{2}{*}{$E_{2010-3010}$} & $E_{11}$ reinforcement corrosion & Medium & Large & 3.88 & \multirow{2}{*}{ Medium } & 3.44 & \multirow{2}{*}{3.36} \\
\hline & $E_{12}$ spalling & Medium & Medium & 4.50 & & 4.13 & \\
\hline \multirow{2}{*}{$E_{1020-2020}$} & $E_{11}$ reinforcement corrosion & Small & Medium & 5.12 & \multirow{2}{*}{ Large } & 4.50 & \multirow{2}{*}{4.37} \\
\hline & $E_{12}$ spalling & Small & Slightly small & 5.62 & & 4.94 & \\
\hline \multirow{2}{*}{$E_{2020-3020}$} & $E_{11}$ reinforcement corrosion & Small & Medium & 5.12 & \multirow{2}{*}{ Medium } & 4.69 & \multirow{2}{*}{4.54} \\
\hline & $E_{12}$ spalling & Small & Slightly small & 5.62 & & 5.06 & \\
\hline \multirow{2}{*}{$E_{1030-2030}$} & $E_{11}$ reinforcement corrosion & Large & Very large & 3.00 & \multirow{2}{*}{ Large } & 2.50 & \multirow{2}{*}{2.31} \\
\hline & $E_{12}$ spalling & Large & Large & 3.25 & & 2.75 & \\
\hline \multirow{2}{*}{$E_{2030-3030}$} & $E_{11}$ reinforcement corrosion & Large & Very large & 3.00 & \multirow{2}{*}{ Medium } & 2.69 & \multirow{2}{*}{2.47} \\
\hline & $E_{12}$ spalling & Large & Large & 3.25 & & 2.88 & \\
\hline
\end{tabular}

TABLE 13: Results beams.

\begin{tabular}{|c|c|c|c|c|c|c|c|}
\hline$E_{i}$ & $E_{i j}$ & $G_{i j k}$ & $W_{i j k l}$ & $n$ & $W E_{i j k l m}$ & $n$ & $n$ final \\
\hline \multirow{2}{*}{$E_{2010-2020}$} & $E_{11}$ shear stress & Small & Medium & 5.12 & \multirow{2}{*}{ Small } & 5.12 & \multirow{2}{*}{5.00} \\
\hline & $E_{12}$ longitudinal stress & Small & Slightly small & 5.62 & & 5.62 & \\
\hline \multirow{2}{*}{$E_{2020-2030}$} & $E_{11}$ shear stress & Medium & Large & 3.88 & \multirow{2}{*}{ Small } & 3.88 & \multirow{2}{*}{3.87} \\
\hline & $E_{12}$ longitudinal stress & Small & Slightly small & 5.62 & & 5.62 & \\
\hline \multirow{2}{*}{$E_{3010-3020}$} & $E_{11}$ shear stress & Medium & Very large & 3.88 & \multirow{2}{*}{ Small } & 3.88 & \multirow{2}{*}{3.79} \\
\hline & $E_{12}$ longitudinal stress & Medium & Large & 4.50 & & 4.50 & \\
\hline \multirow{2}{*}{$E_{3020-3030}$} & $E_{11}$ shear stress & Medium & Large & 3.88 & \multirow{2}{*}{ Small } & 3.88 & \multirow{2}{*}{3.16} \\
\hline & $E_{12}$ longitudinal stress & Large & Large & 3.25 & & 3.25 & \\
\hline
\end{tabular}

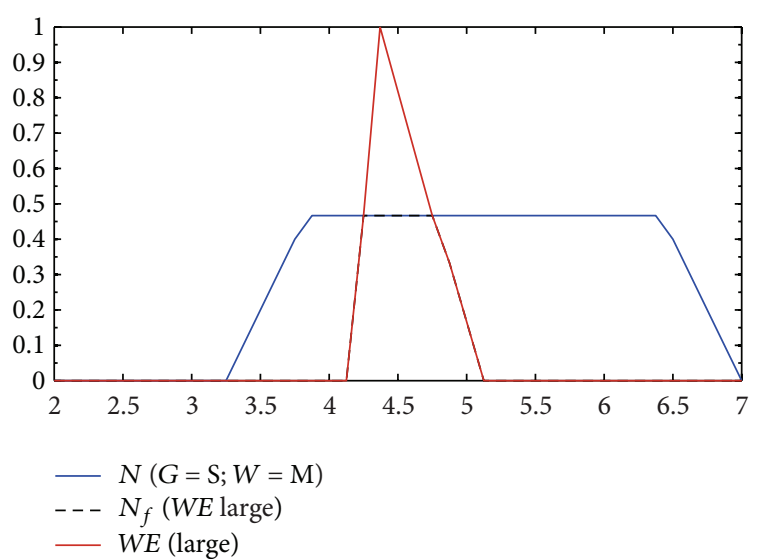

FIGURE 18: Safety exponent achieved by giving $G_{i j k}=$ small, $W_{i j k l}=$ medium, and $W E_{i j k l m}=$ large.

(large to the lower floor columns and medium to the upper floor columns) affected the final safety factor. The results obtained for the row of pilasters 1010-2010-3010, for the row of pilasters 1020-2020-3020, for the row of pilasters 1030-20303030 , and finally for the beams, with associated weight small, are shown (Figure 3).

The choice of the weights was made using the following criteria: firstly the weight of columns of the ground floor is greater than the weight of the other elements of the structure and secondly a greater weight to the columns in respect to the beams is assigned and, within this differentiation, it is necessary to give greater weight to events related to shear degradation rather than to those related to bending.

8.1. Case 1: 1010-2010-3010 Row of Pilasters. For the lower column $\mathrm{E}_{1010-2010}$ to both manifestations of degradation the linguistic subjective judgment medium was assigned obtaining, respectively, $n=3.88$ and $n=4.5$ (the values are different because the weight that each manifestation of degradation has on safety is different, in particular large and medium, resp.).

The same linguistic judgment was given to the higher columns $\mathrm{E}_{2010-3010}$ and accordingly the same results $n=3.88$ and $n=4.5$ were obtained (Table 12 ).

The difference lies in the fact that the weight associated with the element $\mathrm{E}_{1010-2010}$ is large, while the weight associated with the element $\mathrm{E}_{2010-3010}$ is medium. These weights change, according to the fuzzy logic method, the value of the safety exponent associated with each manifestation of degradation, resulting for the structural element $\mathrm{E}_{1010-2010}$ in weighed safety factors, respectively, equal to 3.25 and 3.88 (Table 12), while for the element $\mathrm{E}_{2010-3010}$ we obtain, respectively, a weighted safety factor equal to 3.44 and 4.13 (Table 12).

It is clear then that both the weight large and the weight medium reduce the safety factor $n$ resulting in 
a different safety factor that depends on the weight that the structural element has within the hyperstatic structure. It is important to underline that the unweighted safety factor $n=3.88$ associated with the expression of deterioration under consideration (reinforcement corrosion) is close to the safety range limit equal to 4 and it is furthermore reduced to the value of 3.25 for the structural element $E_{1010-2010}$ and to the value of 3.44 for $\mathrm{E}_{2010-3010}$. Hence the different weight values further reduce the safety coefficient $n$, in the interest of safety. In the same way the unweighted safety coefficient $n=4.5$ associated with the other expression of degradation (detachment of the concrete cover) is reduced to the value 3.88 for the structural element $\mathrm{E}_{1010-2010}$, while for the element $\mathrm{E}_{2010-3010}$ it is reduced to the value 4.13. The safety condition is no longer satisfied when $n<4$.

The fuzzy procedure that relates the safety factor to the weight that the structural element has within the structure further reduces the coefficient within a range determined a priori by experts. In fact, attributing the weight large to both manifestations of degradation safety requirements are no longer satisfied in both cases, while attributing the weight medium safety condition is verified only in the case of the manifestation of degradation spalling.

8.2. Case 2: 1020-2020-3020 Row of Pilasters. In the case under study, to the elements $\mathrm{E}_{1020-2020}$ and $\mathrm{E}_{2020-2030}$ and for both degradation events the linguistic judgment small was assigned, obtaining, respectively, $n=5.12$ and $n=$ 5.62 (Table 12) (different values of the safety coefficients are expected because the weight that each event has on the degradation of safety is different, medium and slightly small, resp.).

The weight $W E$ associated with the element $\mathrm{E}_{1020-2020}$ is large, while the weight associated with $\mathrm{E}_{2020-3020}$ is medium. The result is that the structural element $\mathrm{E}_{1020-2020}$ has weighed safety factors equal to 4.50 and 4.94, respectively (Table 12), while the corresponding values for the element $\mathrm{E}_{2020-3020}$ are 4.69 and 5.06 (Table 12).

Also in this case both the weights large and medium reduce the safety coefficient $n$ obtaining different safety coefficients which depend on the weight that the structural element has in the hyperstatic structure. It should be noted that although the coefficient $n$ is reduced, it is still in a condition of safety because, for both elements, the final result is greater than the safety limit $(n=4)$.

8.3. Case 3: 1030-2030-3030 Row of Pilasters. In this case both $\mathrm{E}_{3010-3020}$ and $\mathrm{E}_{3020-3030}$ and for both manifestations of degradation the linguistic subjective judgment large was assigned obtaining, respectively, $n=3.00$ and $n=3.25$ (Table 12) (the weight that each event has on the degradation of safety is different, resp., very large and large).

The weight $W E$ associated with the element $\mathrm{E}_{3010-3020}$ is large, while the weight associated with the $\mathrm{E}_{3020-3030}$ is medium. As a result for the structural element $\mathrm{E}_{3010-3020}$ for the weighed safety factors the values of 2.50 and 2.75 were obtained (Table 12), while for the element $\mathrm{E}_{3020-3030} 2.69$ and 2.88 were obtained (Table 12 ).
Also in this case both the weight large and the weight medium reduce the value of $n$, obtaining a safety coefficient which depends on the different weight that each structural element has in the hyperstatic structure.

These values lead to an unsafe condition alert for the structural element. The weight $W E$ that the structural element has on the whole structure, respectively, large and medium, further reduces the safety coefficient $n$.

8.4. Results for the Beams. The weight WE associated with the beams is small. In the capacity design the weight $W E$ of the beams is less than the weight of the columns of the ground floor (large) and the first floor (medium).

The attribution of weights medium and large to the structural element reduces the safety factor $n$. This reduction is due to the importance of the element in the mechanism of collapse.

The value of $n$ of the structural element with weight $W E$ small depends on the weight that the manifestation of degradation has on the element (Table 13).

\section{Results of the Application to an Isostatic Structure}

The use of fuzzy logic has allowed an objective result to be obtained, that is, judgment, which is influenced not only by the weight of the manifestation of degradation but also by its localization within the structure. This method was applied to a bridge placed along a road, the SS 195 in Cagliari (Italy), suffering from forms of degradation related to the ULS. The aim of this study is to manipulate through fuzzy logic the subjective linguistic judgments expressed by the inspecting staff on the visual signs of degradation in order to assess the current safety level of the deck of the bridge [15].

In the procedure it was decided to give simple linguistic judgements on the level of degradation and not to make any measurements because often, as in the case under examination, the structural elements are not easy to reach. The presence of water under the bridge physically prevents the measurements of the damage, at least not without considerable cost.

The manifestations of degradation common to beams $E_{i}$ are as follows: corrosion of the longitudinal lower bars $E_{i 2}$ and the consequent detachment of the concrete cover $E_{i 1}$ (two manifestations of degradation for the element $E_{i}$ ). These manifestations are present, in some elements, over the entire length of the beam, while in other places they are located in areas of limited extension.

The corrosion of the lower longitudinal bars, in this case, has different weight when localized at the middle rather than at the ends. Indeed, in a simply supported beam (static scheme of the present case) the kinematic collapse is unique and it occurs when a plastic hinge is formed in the middle. From here comes the need to assess the phenomenon of corrosion whereas the beam is divided into three parts: the centerline and the two left and right ends, consequently giving a different weight to the event itself, depending on its location. 
TABLE 14: Degradation assessment card middle beam and exponent and safety.

\begin{tabular}{lccccc}
\hline Type of degradation $E_{i j}$ & Linguistic judgement $G_{i j k}$ & Safety goal $W_{i j k l}$ & & Safety exponent $n$ \\
\hline \multirow{3}{*}{$E_{i 1}$ spalling } & Small & $G_{i 11}$ & Small & $W_{i 112}$ & 5.75 \\
& Medium & $G_{i 12}$ & Slightly small & $W_{i 123}$ & 4.62 \\
& Large & $G_{i 13}$ & Medium & $W_{i 134}$ & 3.88 \\
\hline \multirow{3}{*}{$E_{i 2}$ reinforcement corrosion } & Small & $G_{i 21}$ & Medium & $W_{i 214}$ & 5.12 \\
& Medium & $G_{i 22}$ & Slightly large & $W_{i 225}$ & $W_{i 236}$ \\
& Large & $G_{i 23}$ & Large & & 3.38 \\
\hline
\end{tabular}

TABLE 15: Degradation assessment card beam ends and exponent and safety.

\begin{tabular}{|c|c|c|c|c|c|}
\hline \multirow[t]{2}{*}{ Type of degradation $E_{i j}$} & \multicolumn{2}{|c|}{ Linguistic judgement $G_{i j k}$} & \multicolumn{2}{|c|}{ Safety goal $W_{i j k l}$} & \multirow{2}{*}{$\frac{\text { Safety exponent } n}{5.75}$} \\
\hline & Small & $G_{i 11}$ & Small & $W_{i 112}$ & \\
\hline \multirow[t]{3}{*}{$E_{i 1}$ spalling } & Medium & $G_{i 12}$ & Slightly small & $W_{i 123}$ & 4.62 \\
\hline & Large & $G_{i 13}$ & Medium & $W_{i 134}$ & 3.88 \\
\hline & Small & $G_{i 21}$ & Slightly small & $W_{i 213}$ & 5.62 \\
\hline \multirow[t]{2}{*}{$E_{i 2}$ reinforcement corrosion } & Medium & $G_{i 22}$ & Medium & $W_{i 224}$ & 4.50 \\
\hline & Large & $G_{i 23}$ & Slightly large & $W_{i 235}$ & 3.50 \\
\hline
\end{tabular}

Such manifestations could affect the bearing capacity of the structural element by reducing the resistant section and for this reason are defined as ULS [23].

Table 14, referred to the centerline, and Table 15, referred to the end, show, for each type of degradation $E_{i j}$, the subjective linguistic judgment $G_{i j k}$ that the operator can choose for each beam $E_{i}$, the weight $W_{i j k l}$ that this judgment has on current safety of the structural element, and the resulting value of the exponent of safety $n$ obtained after inputting the data into the authors' software.

With the application of the proposed method, the visual inspection is "translated" into an objective examination of the state of deterioration of the structures under investigation. The numerical evaluation of the safety factor $n$ allows the safety operators to program targeted maintenance and in severe cases, which may involve a structural risk, perform more detailed investigations and consequently to set priorities of due actions. The peculiarity of the proposed method resides in separating the subjective aspect of the linguistic judgement of the gravity of the damage $G_{i j k}$ from the objective aspect, which is the weight $W_{i j k l}$ that the damage has on safety. It is difficult to have control over the level of knowledge and expertise of the technicians assigned to visual inspections, since these qualities in practice are not homogeneous among the operators as it would be wished for.

\section{Conclusions}

In this paper a procedure suitable for the manipulation and interpretation of subjective linguistic judgments was developed resulting from a visual inspection of a construction, in order to assess, in a more objective and reliable way, the safety level of the building under examination.

With the application of the proposed method, the visual inspection is "translated" into an objective examination of the state of deterioration of the structures under investigation. The numerical evaluation of the safety factor $n$ allows safety inspector to program targeted maintenance and in severe cases, which may involve a structural risk, perform more detailed investigations and consequently to set priorities of due actions. The peculiarity of the proposed method resides in separating the subjective aspect of the linguistic judgement of the gravity of the damage $G_{i j k}$ from the objective aspect, which is the weight $W_{i j k l}$ that the damage has on the safety and the weight $W E_{i j k l m}$ that the element has within the structure. This method provides a quick, low cost, and reliable way of assessing the evolution of degradation in a building.

All the information coming from the visual inspections is managed, evaluated, and processed, thus realizing a management information system based on the authors' software.

Software is developed by the authors which implements their fuzzy logic procedure applied to lexical subjective judgements. The evolution of this procedure described in this paper not only considers the weight associated with the manifestation of degradation but also takes into consideration the weight that each element has on overall structural safety. This allows a specific assessment of detail and a broader assessment of the condition of the building as a whole taking into account the morphology of the structural system.

Interesting results were obtained in the applications described in the previous publications [12-15].

In this way, the costs resulting from this type of investigation are relatively low with no detriment on the reliability of the result which is reflected in the value of the safety factor $n$.

This information management system allows safety operators to sort the data resulting from visual inspection of the structures even when structural number is relevant. For each structure under study, the authors' system returns the safety factor $n$ for each structural element.

The goal of the proposed method is to evaluate and manage all the information coming from visual inspections. In this way, experts can, through the evaluation of the safety factor $n$, assess with homogenous criteria the state of conservation of the structures under investigation, so that 
within a facility or across multiple facilities a hierarchy of interventions can be created.

\section{Conflict of Interests}

The authors declare that there is no conflict of interests regarding the publication of this paper.

\section{References}

[1] D. M. Frangopol, "Life-cycle performance, management, and optimisation of structural systems under uncertainty: accomplishments and challenges," Structure and Infrastructure Engineering, vol. 7, no. 6, pp. 389-413, 2011.

[2] L. Bertolini, Materiali Da Costruzione-Volume II: Degrado, Prevenzione, Diagnosi, Restauro, Città Studi Edizioni, Torino, Italy, 2006.

[3] L. Coppola, "La diagnosi del degrado delle strutture in calcestruzzo," L'Industria Italiana del Cemento, vol. 681, pp. 657-666, 1993.

[4] L. A. Zadeh, "Fuzzy sets," Information and Control, vol. 8, no. 3, pp. 338-353, 1965.

[5] V. I Carbone, G. Mancini, and F. Tondolo, "Structural safety evaluation by means of fuzzy-probabilistic approach," in Proceedings of the 29th Conference on Our World in Concrete \& Structures, Singapore, August 2004.

[6] D. I. Blockley, "Predicting the likelihood of structural accidents," Proceedings. Part 2 Research and Theory, vol. 59, no. 2, pp. 659-668, 1975.

[7] D. Blockley, The Nature of Structural Design and Safety, Ellis Horwood Limited, Chichester, UK, 1980.

[8] B. Kosko, Neural Networks and Fuzzy Systems. System Approach to Machine Intelligence, Prentice Hall, Englewood Cliffs, NJ, USA, 1992.

[9] B. Kosko, Fuzzy Engineering, Prentice Hall, Englewood Cliffs, NJ, USA, 1997.

[10] C. von Altrock, Fuzzy Logic and Neurofuzzy Applications Explained, Prentice Hall, Englewood Cliffs, NJ, USA, 1995.

[11] H. T. Lefteri, Fuzzy and Neural Approaches in Engineering, John Wiley \& Sons, New York, NY, USA, 1997.

[12] M. V. Piras, L. Deias, and F. Mistretta, "Fuzzy logic application to the vulnerability assessment of concrete structures affected by degradation," in Proceedings of the International Conference on Inspection, Appraisal, Repairs and Maintenance of Structures, Yantai, China, April 2010.

[13] M. V. Piras, L. Deias, and F. Mistretta, "Vulnerability analysis of a reinforced concrete structure by visual inspection," in Proceedings of the 5th International Conference on Bridge Maintenance, Safety and Management (IABMAS '10), pp. 20372042, Philadelphia, Pa, USA, July 2010.

[14] M. V. Piras, L. Deias, and F. Mistretta, "Vulnerability assessment of a concrete bridge through fuzzy logic analysis of visual inspection compared to chemical inspection," in Proceedings of the International Association for Bridge and Structural Engineering Symposium (IABSE '10), Venice, Italy, September 2010.

[15] B. De Nicolo, D. Meloni, F. Mistretta, and M. V. Piras, "Inspection based approach for security assessment concrete bridges," in Proceedings of the 12th International Conference on Structural Faults \& Repair 2010, Edinburgh, UK, June 2010.
[16] B. Möller, W. Graf, and M. Beer, "Fuzzy structural analysis using $\alpha$-level optimization," Computational Mechanics, vol. 26, no. 6, pp. 547-565, 2000.

[17] B. Möller, W. Graf, and M. Beer, "Safety assessment of structures in view of fuzzy randomness," Computers and Structures, vol. 81, no. 15, pp. 1567-1582, 2003.

[18] B. Möller, W. Graf, and M. Beer, "Structural reliability analysis through fuzzy number approach, with application to stability," Computers and Structures, vol. 82, pp. 325-327, 2004.

[19] M. Beer, B. Möller, and M. Liebscher, "Processing uncertainty in structural analysis, design and safety assessment," in Proceedings of the 4th International Symposium on Uncertainty Modeling and Analysis (ISUMA '03), B. M. Ayyub and N. O. Attoh-Okine, Eds., pp. 34-39, IEEE, College Park, Md, USA, September 2003.

[20] M. Beer, "Uncertain structural design based on nonlinear fuzzy analysis," ZAMM, vol. 84, no. 10-11, pp. 740-753, 2004.

[21] M. Acito, "Misura Fuzzy della vulnerabilità di manufatti stradali e ferroviari in c.a. interessati da degrado," in $13^{\circ}$ Congresso CTE, Pisa, Italy, Novembre 2000.

[22] UNI EN 1990, "Eurocode basis of structural design,” Tech. Rep. CEN/TC 250, Maggio, 2004.

[23] CEB, Model Code for Service Life Design, Bulletin fib No. 34, 2006.

[24] F. Biondini, F. Bontempi, D. M. Frangopol, and P. G. Malerba, "Cellular automata approach to durability analysis of concrete structures in aggressive environments," Journal of Structural Engineering, vol. 130, no. 11, pp. 1724-1737, 2004.

[25] F. Biondini, F. Bontempi, D. M. Frangopol, and P. G. Malerba, "Probabilistic service life assessment and maintenance planning of concrete structures," Journal of Structural Engineering, vol. 132, no. 5, pp. 810-825, 2006.

[26] F. Biondini, F. Bontempi, D. M. Frangopol, and P. G. Malerba, "Time-variant structural performance of the certosa cablestayed bridge," Structural Engineering International, vol. 16, no. 3, pp. 235-244, 2006.

[27] F. Biondini, D. M. Frangopol, S. Restelli, and F. Biondini, "On structural robustness, redundancy and static indeterminacy," in Structures Congress, pp. 1-10, 2008.

[28] Ch. Massonet and M. Save, Calcul Plastique des Constructions: Structures Depandant d'un Parameter, Edition NELISSEN, Liège, Belgium, 1980.

[29] "NCT2008-Norme tecniche per le costruzioni," D.M. 14, Gennaio, 2008.

[30] UNI EN 1998, Design of Structures for Earthquake Resistance, Part 1: General Rules, Seismic Actions and Rules for Building, 2005.

[31] M. Bruson and S. Martinello, CIAS. Valutazione della capacità portante di un ponte, 4 EMME Service S.p.A, Varese, Italy, 2011.

[32] A. B. Tee, M. D. Bowman, and K. C. Sinha, "A fuzzy mathematical approach for bridge condition evaluation," Civil Engineering Systems, vol. 5, no. 1, pp. 17-24, 1988.

[33] A. Migliacci, "La sicurezza strutturale," in Ingegneria delle strutture, E. Giangreco, Ed., vol. 2, pp. 654-713, Utet, Torino, Italy, 2002.

[34] P. E. Pinto, Elementi di Analisi Probabilistica della Sicurezza, 1972. 

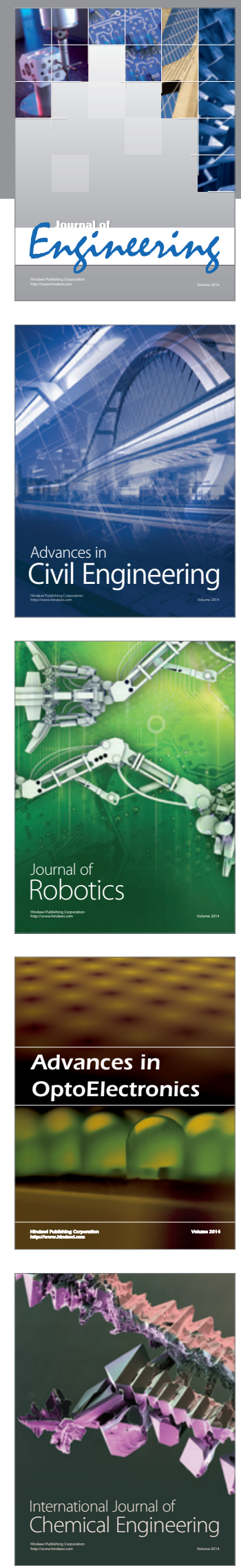

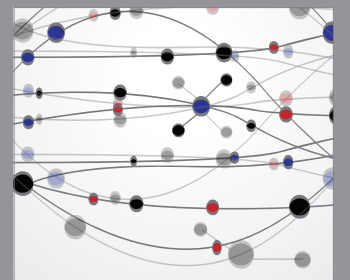

The Scientific World Journal
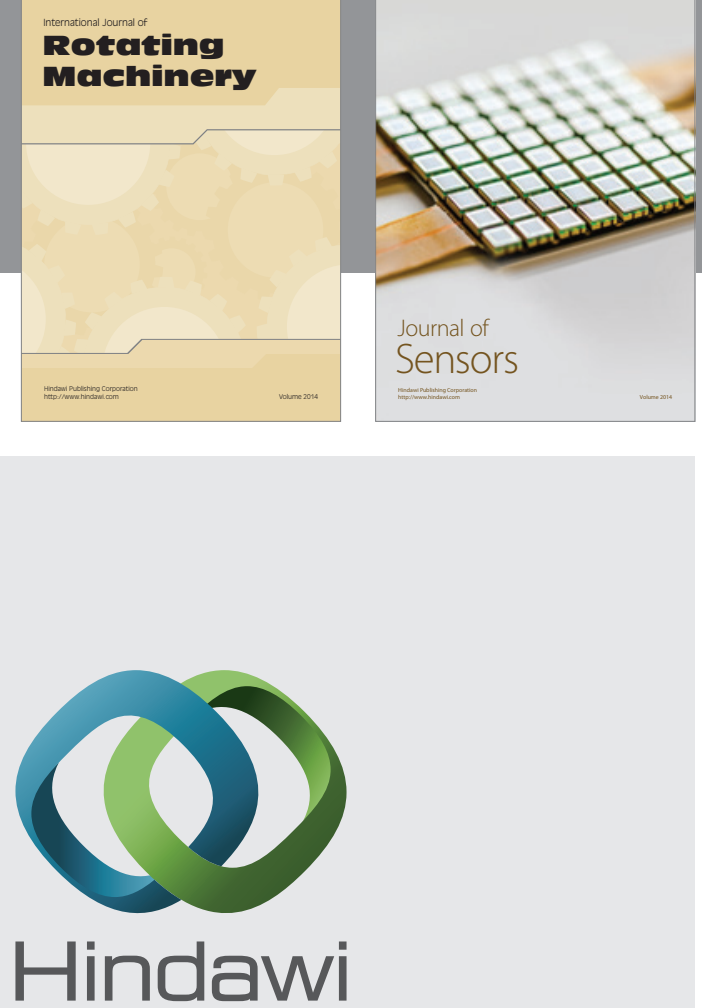

Submit your manuscripts at http://www.hindawi.com
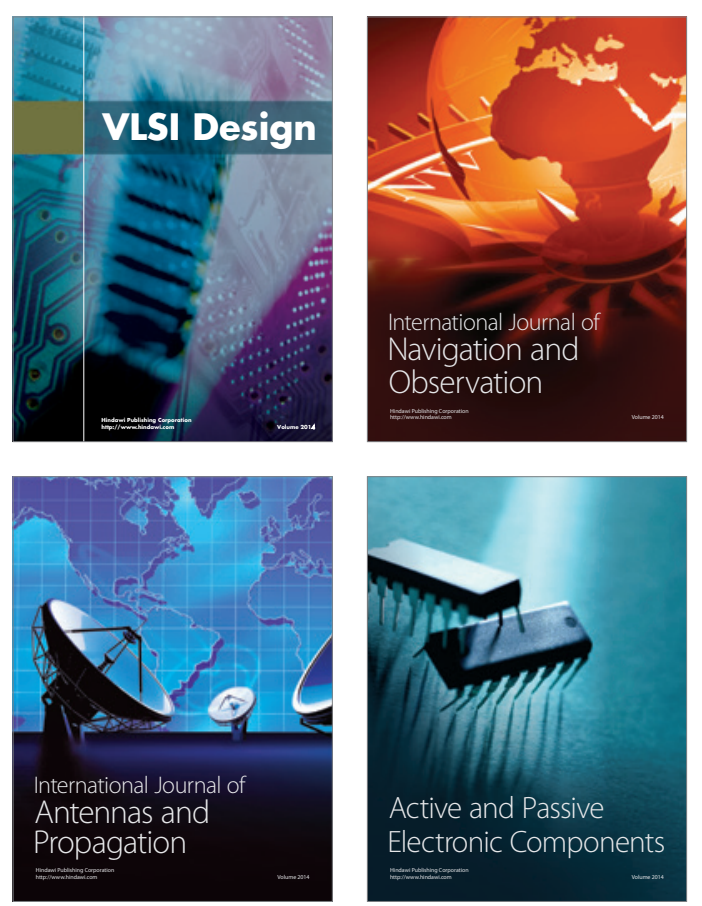
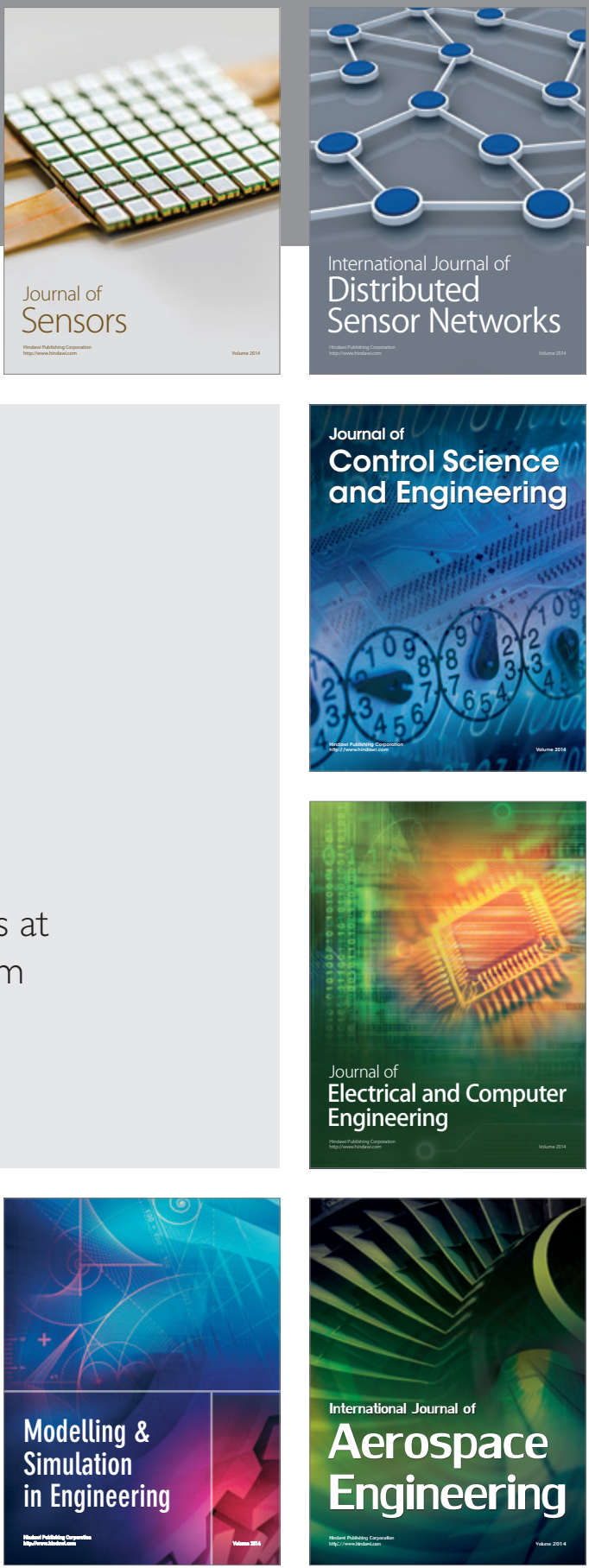

Journal of

Control Science

and Engineering
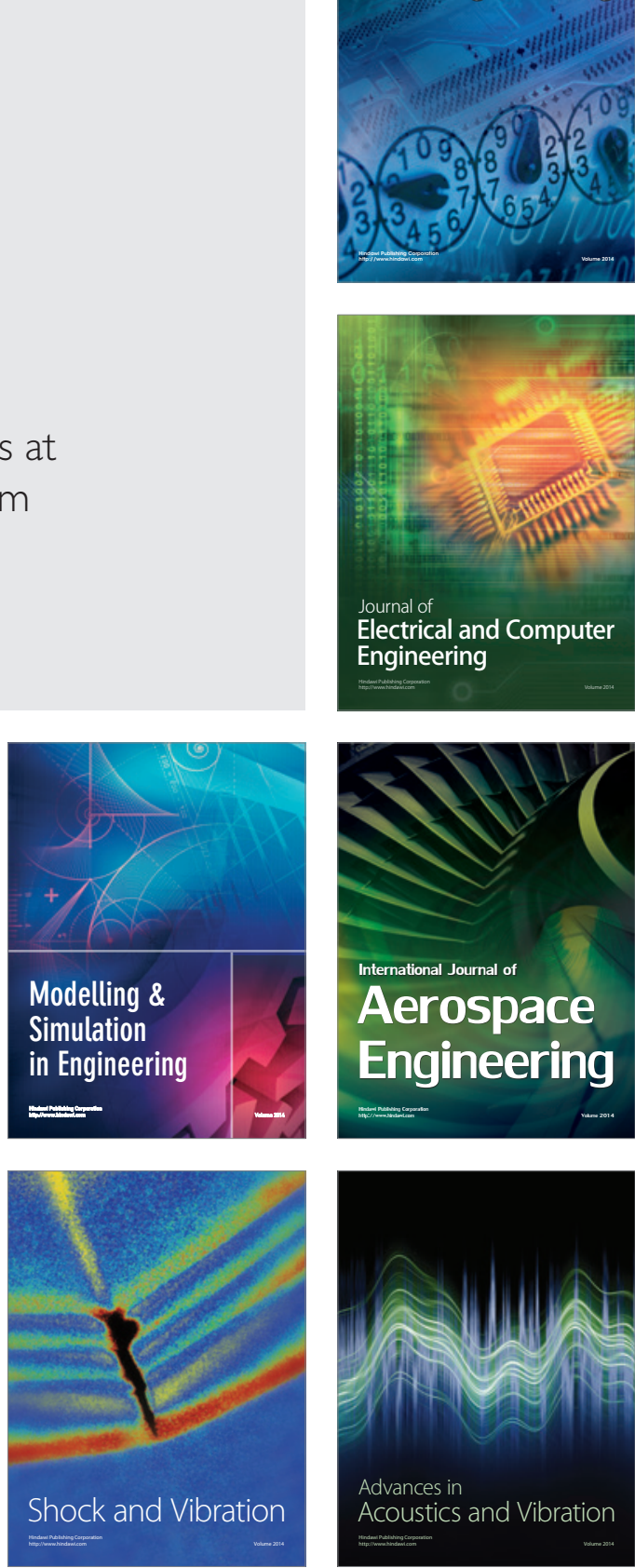